

\title{
Schwere Blutungen an Extremitäten So lassen sie sich stoppen
}

\author{
Autounfall, mit der Motorsäge abgerutscht, vom Stahlträger eingeklemmt: \\ Die Gründe für massiv blutende Wunden an Armen und Beinen sind viel- \\ fältig. Zum Glück kommen solche Verletzungen selten vor - und sie lassen \\ sich sogar vergleichsweise gut erstversorgen. Hier stellen wir anhand eines \\ Fallbeispiels die algorithmenbasierte Versorgung bei der kritischen Blutung \\ einer Extremitätenverletzung dar.
}

Eher seltener Einsatzgrund Kritische Blutungen von Extremitäten sind im Notarzt- und Rettungsdienst eher selten anzutreffen [1]. Die Erfahrung mit diesen Verletzungen stammt hauptsächlich aus Kriegen: Fast die Hälfte der Opfer (46\%) verstirbt an katastrophalen Blutungen. Davon betreffen jedoch $80 \%$ den Körperstamm - und „nur“ 20\% Blutungen aus Extremitäten [2]. Im zivilen Alltag begegnen einem lebensbedrohliche Extremitätenblutungen u.a. bei Arbeitsunfällen, wie auch das folgende Beispiel illustriert.

Beispiel: Arbeitsunfall mit Eisenträger Ein 22-jähriger Arbeiter wird in $4 \mathrm{~m}$ Höhe auf einer Stahlkonstruktion von einem tonnenschweren Träger ( $\bullet$ Abb. 1) am rechten Unterschenkel getroffen.

- Er erleidet eine stark blutende, drittgradig offene Unterschenkelfraktur.

Anwesende Kollegen retten ihn von der Hallenkonstruktion, er zieht sich keine weiteren Verletzungen zu. Als der Rettungsdienst eintrifft (NEF und RTW), liegt er am Fuße der Hallenkonstruktion.

- Der rechte Unterschenkel ist offensichtlich fehlgestellt und blutet stark ( $\odot$ Abb. 2).

Nach kurzer Lageerkundung steht fest, dass keine akute weitere Gefahr für das Team besteht: Der Stahlträger ist fest angeschlagen und durch einen Kran gesichert, es kann gefahrlos gearbeitet werden. Weitere Gefahren an der Einsatzstelle bestehen nicht.

- Das Team kümmert sich nun um den Patienten. Eigen- und fremdanamnestisch können weitere Verletzungen ausgeschlossen werden.

- Auffallend ist die starke Blutung aus der Unterschenkelwunde. Der Patient zeigt die klinischen Zeichen eines Volumenmangelschocks.

Wie sieht ein mögliches Vorgehen des Rettungsteams aus?

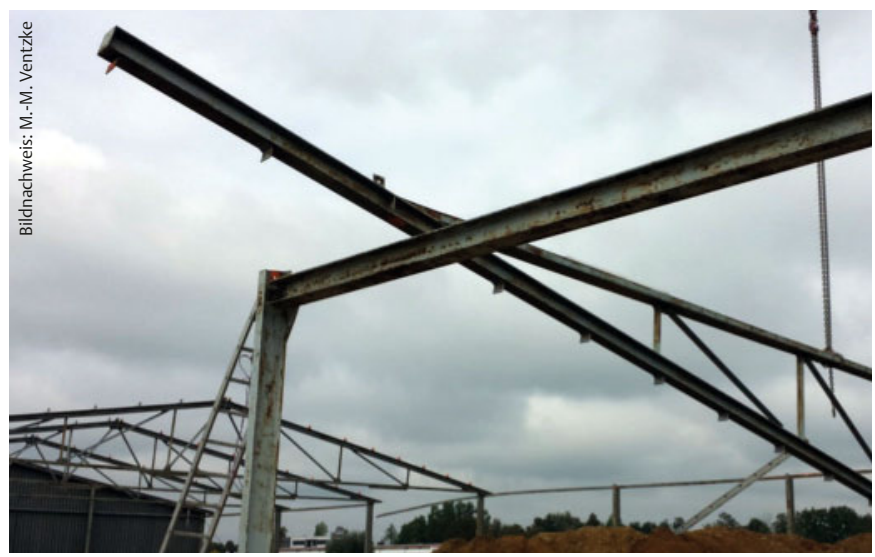

Abb. 1 Blick von unten auf den Dachträger, der umgeschlagen ist und den in $4 \mathrm{~m}$ Höhe auf dem Querträger sitzenden Patienten am Unterschenkel getroffen hat.

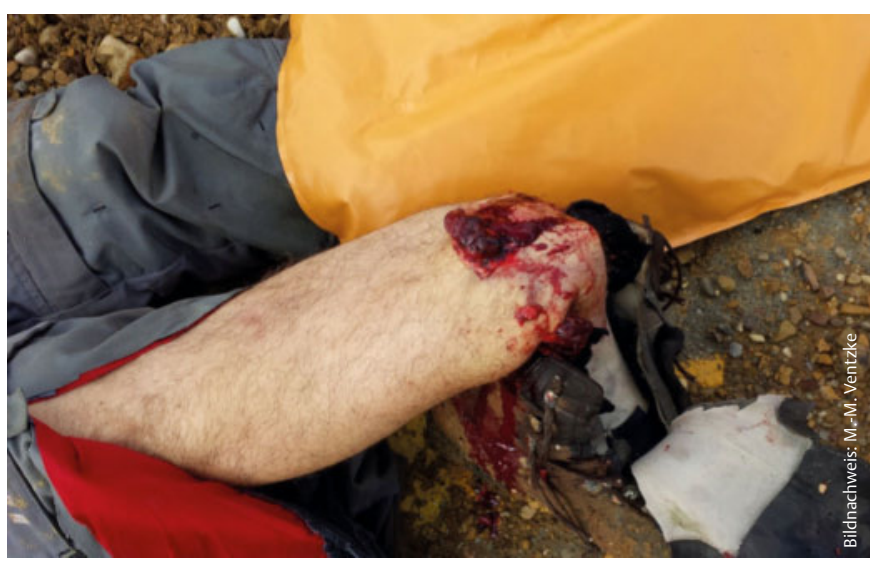

Abb. 2 Drittgradig offene Unterschenkelfraktur rechts mit starker Blutung. Ein Großteil des verlorenen Blutes ist im Bild verdeckt. Auffällig: die verblasste, nicht mehr durchblutete Extremität distal der Fraktur (im Stiefel). 
Wodurch entsteht eine TIC?

Nach einem schweren Trauma kommt es schon innerhalb von Minuten zu Gerinnungsstörungen. Grund dafür sind der Verbrauch von Gerinnungsfaktoren und die Hemmung verschiedener Schritte der Hämostase. Das schnelle Auftreten dieser traumainduzierten Koagulopathie geht dabei auf folgende Faktoren zurück:

- Gewebeverletzung

- Entzündung

- Minderperfusion/Schock

- Verdünnung/Verlust von Gerinnungsfaktoren und Erythrozyten (auch durch Infusionslösungen)

- Hypothermie

- Azidose

\section{Was lässt sich präklinisch tun?}

- 1. Blutung stillen Stoppen Sie Blutverluste sofort und vordringlich, auch bei einem Massenanfall von Verletzten.

- 2. angepasste Volumengabe Ziel ist es, die Gewebeperfusion aufrechtzuerhalten, ohne die Gerinnungsfaktoren unnötig zu verdünnen. Dabei muss man keine Blutdruck-Normwerte erzielen (sog. permissive Hypotension mit $R_{\text {syst }} \approx 90 \mathrm{mmHg}$ ). Ausnahmen sind Patienten mit schwerem Schädel-Hirn-Trauma, ggf. auch mit Wirbelsäulenverletzungen, und Schwangere (Zielwert hier: $R_{\text {syst }}=110-120 \mathrm{mmHg}$ ).

$\triangleright$ Achtung: Kolloidale Infusionslösungen bleiben länger im Intravasalraum und verdünnen die Gerinnungsfaktoren länger. Zudem setzen sie in großen Mengen die Thrombozytenfunktion und die Thrombusstabilität herab. Wenn Sie balancierte und gepufferte kolloidale und kristalloide Lösungen verwenden, sollte das Verhältnis Kolloid: Kristalloid $<1: 2$ sein.

- 3. keine Hypothermie! Pro $1{ }^{\circ} \mathrm{C}$ Temperaturverlust vermindert sich die Funktion der Gerinnungsfaktoren um $10 \%$. Außerdem werden die Fibrinolyse gesteigert und die Thrombozytenaggregation beeinträchtigt. Temperaturen $<34^{\circ} \mathrm{C}$ sind kritisch - hier ist die Aktivität der Gerinnungsfaktoren deutlich reduziert.

$\triangleright$ Verwenden Sie daher am besten warme Infusionen,

$\triangleright$ entfernen Sie nasse Kleidung,

$\triangleright$ sorgen Sie für schnellen Wärmeerhalt und

$\triangleright$ untersuchen Sie den entkleideten Patienten nach Möglichkeit im (vorgeheizten) RTW.

- 4. Azidose vermeiden Die schockinduzierte Minderperfusion führt zu einer Azidose. Ein Blut-pH-Wert $\leq 7,2$ schränkt die Thrombozytenfunktion und Aktivität von Gerinnungsfaktoren deutlich ein.

$\triangleright$ Hier gilt: Vermeiden und behandeln Sie den Schock, statt blind auf pH-Werte im Normbereich zu puffern. Das wäre „Laborkosmetik“, würde aber Mikrozirkulation und Koagulopathie nicht bessern.

\section{Wichtigstes Prinzip: Prioritäten-orientierte Versorgung des Traumapatienten}

Viele Versorgungskonzepte - ein Ziel Für Traumapatienten existiert eine Vielzahl international anerkannter Versorgungskonzepte (PHTLS: Prehospital Trauma Life Support, ITLS: International Trauma Life Support, ATLS: Advanced Trauma Life Support, TCCC: Tactical Combat Casualty Care u.a.). Ihr Ziel ist es, den Patienten zeitnah - am besten innerhalb $1 \mathrm{~h}$ (sog. „golden hour“) einer chirurgischen Versorgung zuzuführen. Präklinisch kommt es v.a. darauf an,

- den Patienten Prioritäten-orientiert zu behandeln („treat first what kills first") und

- weiteren Schaden vom Patienten abzuwenden („do no further harm“) [3].
ABCDE-Schema dient als Basis Allen gemein ist das sog. ABCDESchema (Airway, Breathing, Circulation, Disability, Exposure), d.h. es erfolgt die Untersuchung und sofortige Behandlung von lebensbedrohlichen Störungen

- A) des Atemwegs (Atemwegsverlegungen und Stabilisierung einer eventuellen Fraktur der Halswirbelsäule (HWS)),

- B) der Atmung (v.a. Spannungspneumothorax),

- C) von kritischen Blutungen,

- D) zentralen neurologischen Störungen (Schädel-Hirn-Trauma) sowie

- E) die komplette Untersuchung und Wärmerhalt.

Traumainduzierte Koagulopathie als zusätzliche Komplikation

Allerdings sind schwere Blutungen beim Trauma oft mit gravierenden Blutgerinnungsstörungen vergesellschaftet. 
- Diese sog. traumainduzierte Koagulopathie (TIC; 0 Infobox S. 196) wird als eigenständiges Krankheitsbild zusätzlich zu den erlittenen Verletzungen beschrieben [5].

Des Weiteren können beim Trauma neben dem Krankheitsbild der TIC noch weitere Blutgerinnungsstörungen auftreten. Dazu gehören:

- Verlustkoagulopathie

- disseminierte intravasale Gerinnung und Verbrauchskoagulopathie

- Verdünnungskoagulopathie

Problem: Gerinnungs- und plättchenhemmende Pharmaka Erschwerend kommt im „zivilen“ Rettungsdienst die Tatsache hinzu, dass viele - und aufgrund der alternden Bevölkerung immer mehr - Patienten mit gerinnungs- und thrombozytenaggregationshemmenden Medikamenten behandelt sind ( $\boldsymbol{0}$ Tab. 1). Deren Wirkung lässt sich im präklinischen Setting i.d.R. nicht aufheben bzw. therapieren - entweder wegen nicht vorgehaltener Gerinnungsfaktoren/-therapeutika oder in Ermangelung möglicher Therapieoptionen.

$<C>A B C D E$ statt ABCDE Die oben genannten Umstände und die Erfahrungen aus militärischen Konflikten [6] führten zum Umdenken bei lebensbedrohlichen Blutungen, sodass dem bekannten PHTLS-Algorithmus ein $<\mathrm{C}>$ für „critical bleeding“ (katastrophale/kritische Blutung) vorangestellt wurde: $<\mathrm{C}>\operatorname{ABCDE}[7,8]$.

- Das bedeutet, dass massive Extremitäten-Blutungen vor der weiteren Patientenversorgung gestoppt werden sollten.

Dieses Vorgehen hat auch Eingang in die deutschen Leitlinien gefunden (S3-Leitlinie Polytrauma / Schwerverletzten-Behandlung), in der es heißt [9]: „Starke und unmittelbar lebensbedrohliche Blutungen sollen sofort versorgt werden, auch unter Vernachlässigung des ABCDE-Schemas.“

\section{So stoppen Sie Extremitätenblutungen}

Abgestufte Vorgehensweise Gemäß der S3-Leitlinie Polytrauma der Deutschen Gesellschaft für Unfallchirurgie kommt bei stark blutenden Extremitätenverletzungen ein Stufenschema zur Anwendung:

- Die betroffene Extremität sollte hochgelagert, die Blutungsstelle manuell komprimiert und im weiteren Verlauf mittels Druckverband versorgt werden.

Interessanterweise sprechen sich die aktuellen Reanimationsleitlinien des European Resuscitation Council (ERC) im Kapitel „Erste Hilfe“ mangels wissenschaftlichen Nachweises gegen ein Abdrücken und Hochlagern aus [10].

\begin{tabular}{lll}
\hline $\begin{array}{l}\text { Tab. } 1 \text { Gängige Thrombozytenaggregationshemmer und } \\
\text { orale Antikoagulanzien }\end{array}$ & Wirkstoff & Handelsname \\
\hline $\begin{array}{l}\text { Thrombozyten- } \\
\text { aggregationshemmer }\end{array}$ & Azetylsalizylsäure & $\begin{array}{l}\text { ASS, Aspirin, Herz-ASS, } \\
\text { u.v. m }\end{array}$ \\
& Clopidogrel & Plavix, Iscover \\
& Prasugrel & Efient \\
& Ticagrelor & Brilique \\
\hline orale Antikoagulanzien & Phenprocoumon & $\begin{array}{l}\text { Marcumar, Phenpro- } \\
\text { gamma, Marcuphen, }\end{array}$ \\
& Falithrom \\
& Apixaban & Eliquis \\
& Edoxaban & Lixiana \\
& Rivaroxaban & Xarelto \\
& Dabigatran & Pradaxa \\
\hline
\end{tabular}

Tab. 2 Indikationen für den sofortigen Einsatz eines Tourniquets im Rahmen von lebensbedrohlichen Extremitätenblutungen (nach [8, 10])

- starke Blutung bei gleichzeitig anderweitig vital bedrohtem Patienten

- eigentliche Verletzung nicht erreichbar/zugänglich

- Abdrücken/manuelle Kompression/Druckverband nicht möglich

- mehrere Blutungen an einer Extremität

- Zeitdruck in Gefahrensituationen (z. B. sofortige Rettung aus Gefahrenbereich)

- Massenanfall an Verletzten

- Kann die Blutung auch mittels fokussiertem Kompressionsverband nicht gestoppt werden, so ist als nächstes das Tourniquet einzusetzen [9].

Bei bestimmten Indikationen ( $\bullet$ Tab. 2) ist auch die initiale (temporäre) Anlage eines Tourniquets sinnvoll und erlaubt [11].

Konkret: Blutstillung im Fallbeispiel Aufgrund der starken Blutung und der klinischen Schockzeichen entscheidet sich der Notarzt im eingangs geschilderten Beispiel als Erstmaßnahme für das Abdrücken der Oberschenkelarterie im Leistenbereich - ein Abdrücken der Kniearterie wäre mit zu viel Bewegung des Bruches einhergegangen. Daraufhin sistiert die Blutung fast komplett. Diese Maßnahme dient jedoch nur vorübergehend der Blutstillung, bis das Team ein Tourniquet am Oberschenkel der verletzten Extremität anlegt - erst danach sistiert die Blutung vollständig.

Die Extremität hochzulagern, ist bei offener, dislozierter Fraktur und starken Schmerzen nicht angezeigt - wäre aber wahrscheinlich auch wenig effektiv.

Das Tourniquet Das Wirkprinzip eines Tourniquets ist simpel:

- Mithilfe eines Gurts bindet man die Extremität so weit ab, bis die verletzten Blutgefäße abgedrückt werden und somit kein Blut mehr austreten kann. 
Die ersten Beschreibungen von Tourniquets reichen bis in die griechische Antike zurück, und auch im alten Rom setzten Chirurgen sie bereits zum Stillen von Blutungen ein. Seit mehreren Jahren erleben sie - aus der Militärmedizin kommend [12] - eine Renaissance im zivilen Rettungsdienst.

- Nach der S3-Leitlinie Polytrauma sollten sie als sichere, schnelle und effektive Methode der Blutungskontrolle bei stark blutenden Extremitätenverletzungen nicht nur als letzte Möglichkeit, sondern sogar routinemäßig eingesetzt werden [9].

- Auf dem Markt existieren verschiedene Tourniquet-Modelle, auch pneumatische zum Aufpumpen (Übersicht in [13]).

Egal, welches Sie verwenden: Wichtig ist, dass Sie sich vor dem Einsatz mit dem vorgehaltenen Modell auseinandersetzen. Nur wenn das Tourniquet richtig angelegt wird, kann es seinen Zweck erfüllen ( $\bullet$ Abb. 3$)$.

Tourniquet richtig anlegen Unabhängig vom Modell sind beim Anlegen eines Tourniquets einige grundsätzliche Dinge zu beachten:

- Das Tourniquet wird mind. $5 \mathrm{~cm}$ proximal der Blutung an der verletzten Extremität direkt auf der Haut angelegt, um ein Abrutschen zu verhindern.

$\triangleright$ Legen Sie es jedoch nicht über Gelenken, Frakturen oder Fremdkörpern an!

- Dann wird es festgezogen, bis die Blutung steht. Da sich hierbei ein starker Ischämieschmerz entwickelt, ist eine suffiziente Analgesie obligat.

- Ob die Maßnahme effektiv war, erkennen Sie primär am Sistieren der Blutung - nicht am distalen Puls [9].

$\triangleright$ Achten Sie insb. darauf, dass der arterielle Blutfluss komplett unterbrochen wird: Ein fehlerhaft angelegtes bzw. zu locker angelegtes Tourniquet kann die Blutung verstärken, wenn nur das venöse System komprimiert wird! Eine regelmäßige Kontrolle ist obligat.

- Positionieren Sie den Knebel bzw. den Verschlussmechanismus des Tourniquets nach Möglichkeit gut sichtbar ventral oder lateral an der Extremität. Dies gewährleistet eine gute Zugänglichkeit, und man kann ein eventuelles unbeabsichtigtes Öffnen erkennen [14].

- Lässt sich die Blutung nicht stoppen, ist das Tourniquet nachzuziehen und ggf. ein zusätzliches proximal davon anzulegen [9].

Fehlt ein Tourniquet, kann man auch eine Blutdruckmanschette verwenden, die man auf suprasystolischen Druck aufpumpt (Oberarm i.d.R. $250 \mathrm{mmHg}$, Oberschenkel $400 \mathrm{mmHg}$ ) [15, 16]. Sichern Sie diese besonders, um ein akzidentelles Öffnen zu verhindern!
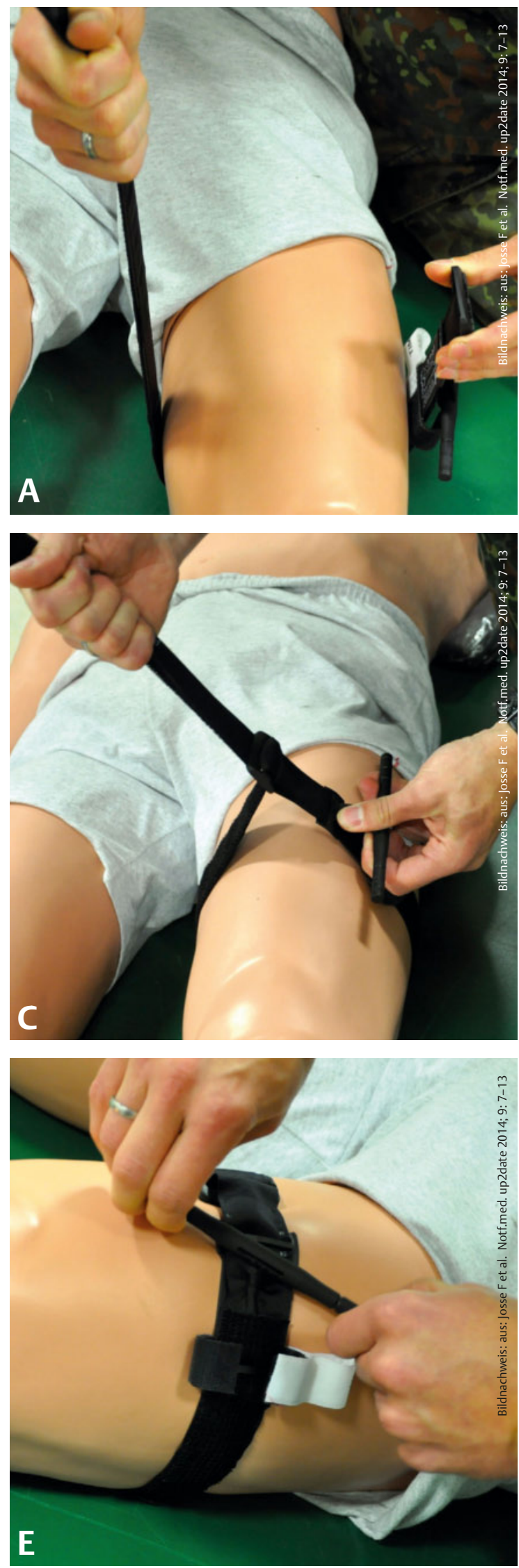

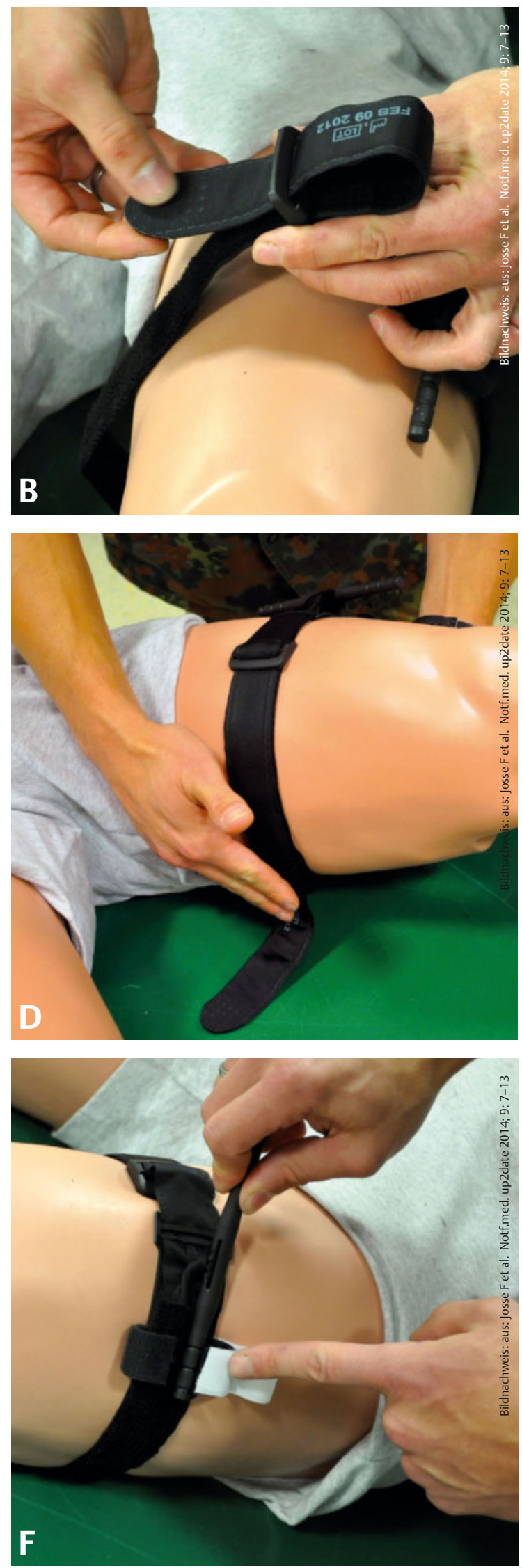

Dokumentation In jedem Fall ist der Anlagezeitpunkt des Tourniquets nachvollziehbar zu dokumentieren und zu übergeben - im Rettungsdienst- / Notarztprotokoll, auf dem Tourniquet selbst, ggf. sogar sichtbar auf dem Patienten [15-17].

Wie lange sollte man ein Tourniquet belassen? Die Datenlage hier$\mathrm{zu}$ ist schwach und es ist unklar, wie lange ein Tourniquet sicher belassen werden kann. Die generelle Empfehlung liegt bei max. $2 \mathrm{~h}$, abgeleitet aus einer Studie von Lee [16]. Ab $2 \mathrm{~h}$ beobachtete Kragh eine höhere Rate an Amputationen und Fasziotomien (s. unten) [18].

- Insgesamt kann man eine Dauer von $2 \mathrm{~h}$ als sicher ansehen [19].

- Die Deutsche Gesellschaft für Unfallchirurgie (DGU) schlägt vor, das Tourniquet zu belassen, sofern die Zeit bis zur operativen Versorgung $<1 \mathrm{~h}$ liegt.

Dies dürfte im zivilen Rettungsdienst i.d. R. zu realisieren sein.

- Bei Rettungszeiten $>1 \mathrm{~h}$ empfiehlt die Fachgesellschaft, das Tourniquet zu lösen und zu überprüfen, ob es zu einer erneuten Blutung kommt. Diese Empfehlung gilt jedoch nur für den stabilisierten Patienten [9]!

Präklinisch wird es normalerweise aber bei dem Vorgehen bleiben, ein einmal angelegtes Tourniquet nicht wieder zu lösen. Diesbezüglich sind sicherlich noch weitergehende Untersuchungen notwendig. Außerdem wird die Deutsche Gesellschaft für Anästhesie in naher Zukunft eine Handlungsempfehlung herausgeben, die bei Transportzeiten $<30$ min und anderen Möglichkeiten der effektiven Blutstillung eine kürzere Zeitspanne zur Konversion des Tourniquets angibt.

\section{Komplikationen durch das Tourniquet eher selten Grundsätzlich} ist die Anlage eines Tourniquets einfach und sicher zu realisieren und die Komplikationsrate gering.
Abb. 3 Richtiges Anlegen eines Tourniquets am Oberschenkel, hier am Beispiel des Combat Application Tourniquet (C.A.T.) [14]. A: Bringen Sie das C.A. T. proximal am Oberschenkel an - mit dem Knebel am lateralen bis ventralen Rand der Extremität. B: Achtung: Bei der Anlage am Oberschenkel muss man das Band durch beide Ösen der Schnalle durchschlaufen. Halten Sie beim Zurückschlaufen die Finger zwischen die beiden Bänder, um ein Verkleben der beiden Klettseiten zu vermeiden (das Lösen kostet Zeit!). C: Ziehen Sie das Tourniquet fest. Dabei ist es wichtig, den Knebel als Gegenpunkt gut an der lateralen Seite des Oberschenkels festzuhalten und mit der anderen Hand das Band kräftig durch die Schnalle zu ziehen, bis es eng an der Haut aufliegt. Es dürfen keine 2 Finger mehr zwischen Haut und Band passen, da sonst kein effektiver Druck durch den Knebel erzeugt werden kann. D: Fixieren Sie das Band, indem Sie das verbleibende Klettband um die Extremität führen und am Band fest andrücken. E: Nun müssen Sie den Knebel des Tourniquets fest zudrehen - die Drehrichtung ist egal. Relevant ist jedoch, dass Sie das Tourniquet so weit anziehen, bis die Blutung zum Stehen kommt. Wichtig: Das Zudrehen des Tourniquets verursacht starke Schmerzen. Schmerzangaben des Patienten dürfen jedoch keinesfalls dazu führen, ein Tourniquet nicht bis zum Sistieren der Blutung „zuzudrehen“. Statt einer Abbindung würde so lediglich eine venöse Stauung mit konsekutiv verstärktem Blutverlust provoziert. Achten Sie deshalb stets auf eine ausreichende Analgesie. F: Ist das Tourniquet ausreichend fest angezogen, dann fixieren Sie den Knebel in einem der beiden u-förmigen Haken und sichern ihn mit dem dafür vorgesehenen weißen Klettband. Hierauf kann man auch die Zeit der Tourniquetanlage dokumentieren. Das Klettband des C.A.T. ist mit dem Wort "Time“ gekennzeichnet. 
- Insbesondere der vielfach gefürchtete Verlust einer Extremität durch den Einsatz eines Tourniquets scheint eher im Bereich der Anekdoten angesiedelt zu sein [20].

Durch die zirkuläre Kompression der Extremität und aller darunter liegender Strukturen sind sicherlich Verletzungen derer denkbar, sodass Haut-, Nerven-, Gefäß- und Muskelschädigungen bis hin zum Kompartmentsyndrom sowie Thromboembolien möglich sind [13]. Eine Analyse der Datenbank des britischen Militärs fand 3 Komplikationen bei 1375 Patienten: 2 Kompartmentsyndrome und 1 Nervenschädigung, die jedoch durch die fehlerhafte Anlage des Tourniquets bedingt waren [21].

- Im Gegensatz dazu steht der Nutzen der Maßnahme: die deutlich größere Überlebenswahrscheinlichkeit bei frühem Einsatz [22].

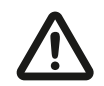

Die Hauptgefahr besteht darin, das Tourniquet ungenügend fest anzuziehen und so durch eine venöse Stauung die Blutung zu verstärken. Daher ist ein regelmäßiges Anwendungstraining wichtig und notwendig.

\section{Blutung gestillt - so geht es weiter}

<C>ABCDE-Schema vervollständigen Nach erfolgreicher Behandlung der kritischen Blutung $(<\mathrm{C}\rangle)$ wendet sich das Team im Fallbeispiel der weiteren algorithmusbasierten Versorgung des Patienten $\mathrm{zu}$ :

- A - Airway und HWS: Der Patient kann genaue Angaben zu Unfallhergang und die Zeit bis zum Eintreffen des Rettungsdienstes machen. Eigen- und fremdanamnestisch lassen sich weitere Verletzungen, insb. eine Verletzung der HWS, ausschließen, sodass auf die Anlage einer HWS-Orthese verzichtet werden kann.

- B - Breathing: Der Thorax des Patienten hebt und senkt sich seitengleich, der Notarzt kann keine Instabilitäten oder Krepitationen tasten, kein Druckschmerz, Vesikuläratmen beidseits, die peripher gemessene Sauerstoffsättigung beträgt $98 \%$ unter Raumluft.

- C - Circulation: Das Abdomen ist unauffällig, das Becken und beide Oberschenkel sind stabil, in der Untersuchung kann das Team keine weiteren relevanten Blutungen feststellen. Bei der Kontrolle der verletzten Extremität steht die Blutung weiterhin (Reevaluation!).

- D - Disability: Der Patient ist neurologisch völlig unauffällig, der Notarzt stellt keinerlei Hinweise auf ein Schädel-HirnTrauma oder Wirbelsäulenverletzungen fest.

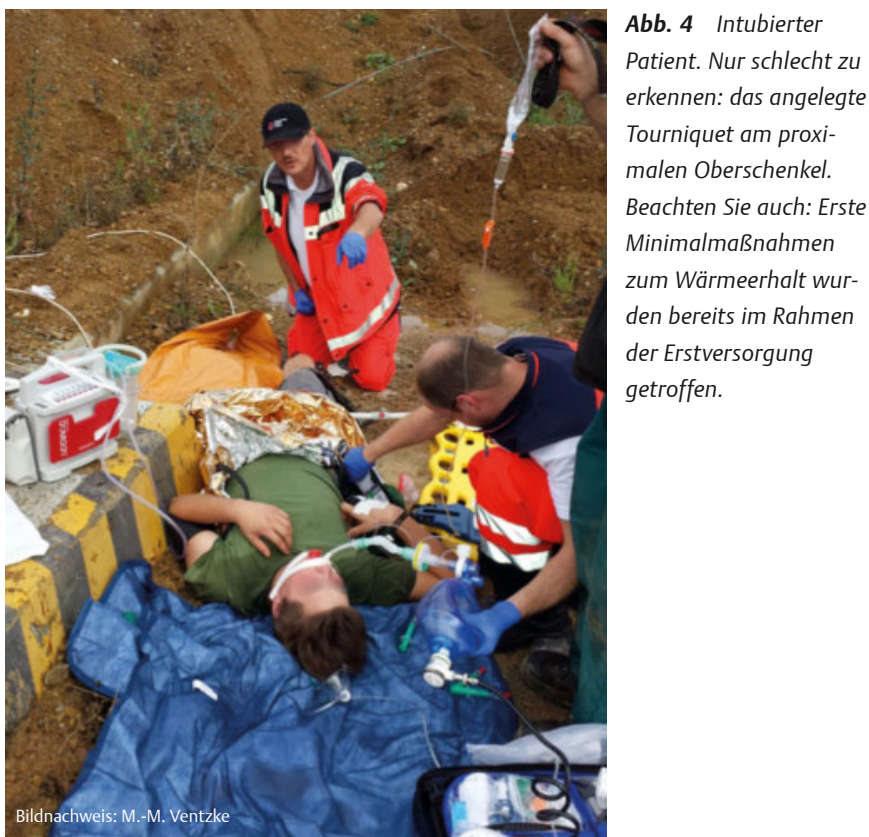

- E - Exposure: Hier kommt dem Wärmeerhalt besondere Bedeutung zu, um die Gerinnungssituation nicht noch durch Wärmeverlust zu verschlechtern. Das Team nutzt dafür Silberfolie und heizt den RTW vor.

Weitere Versorgung unter Narkose Nach Stillen der Blutung und Ausschluss weiterer lebensbedrohlicher Verletzungen entscheidet sich der Notarzt zur präklinischen Narkose und Intubation aus folgenden Gründen:

- suffiziente Analgesie des über massive Schmerzen klagenden Patienten

- erforderliche Reposition der noch fehlgestellten Extremität

- vorhandener Volumenmangelschock

- ohnehin erforderliche operative Versorgung

Während der Vorbereitungen zur Narkoseeinleitung untersucht der Notarzt den Patienten nochmals gründlich auf weitere, bislang nicht entdeckte, nicht lebensbedrohliche Verletzungen. Die Narkoseinduktion gestaltet sich völlig problemlos ( $\triangle \mathrm{Abb} .4$ ), die verletzte Extremität wird achsengerecht reponiert, geschient und die Effektivität des Tourniquets nochmals überprüft. Bei offener Fraktur appliziert das Team bereits präklinisch eine Antibiose. Für den Transport wird der Patient, nachdem die Blutung jetzt steht und nach Infusionstherapie normwertige Blutdruckwerte vorliegen, in flacher Rückenlage gelagert. Der Transport ins Traumazentrum und die Übergabe im Schockraum verlaufen komplikationslos.

Outcome des Patienten Der Patient wird nach sofortiger Schockraum-Versorgung direkt operiert. Wegen der komplexen Gefäßbeteiligung ziehen die Unfallchirurgen sofort einen Gefäßchirurgen 
hinzu. Das verletzte Bein stabilisieren sie zunächst wegen der offenen Fraktur und der schwierigen Weichteilverhältnisse mittels Fixateur interne. Es folgen noch mehrere operative Eingriffe. Letztlich gelingt es wegen der Verletzungsschwere aber nicht, das Sprunggelenk zu erhalten, sodass der Unterschenkel amputiert werden muss.

\section{Fazit}

Kritische Blutungen aus Extremitätenverletzungen sind zwar selten - liegen diese jedoch vor, ist das Vorgehen nach dem bekannten ABCDE-Schema dahingehend abzuändern, dass vordringlich die lebensbedrohlichen Blutungen gemäß dem $\angle C>A B C D E-S c h e m a$ gestoppt werden sollten. Hierzu steht mit dem Tourniquet bei entsprechenden Indikationen eine wirkungsvolle Maßnahme zur Verfügung.

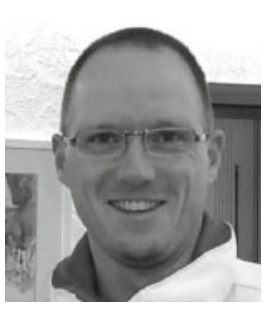

Dr. Marc-Michael Ventzke arbeitet als Facharzt für Anästhesie an der Klinik für Anästhesie und Intensivmedizin, Sektion Notfallmedizin des Bundeswehrkrankenhauses Ulm. Er ist Leitender Notarzt und besitzt langjährige Erfahrung im Boden- und Luftrettungsdienst. Sein Schwerpunkt liegt in der Ausbildung junger Notärzte.

E-Mail: marcmichaelventzke@bundeswehr.org

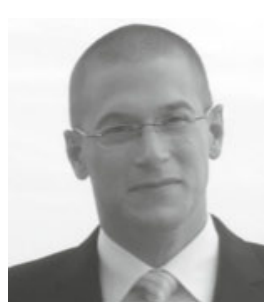

Florent Josse ist Facharzt für Anästhesie und Mitglied der AG Taktische Medizin der DGAI. Er ist verantwortlich für die Ausbildung im Bereich taktische Verwundetenversorgung am Bundeswehrkrankenhaus Ulm, hat mehrfach an Auslandseinsätzen teilgenommen und besitzt langjährige Erfahrung im Boden- und Luftrettungsdienst.

E-Mail: florentjosse@bundeswehr.org

\section{Infos im Internet}

Das Literaturverzeichnis zu diesem Beitrag finden Sie im Internet: Rufen Sie unter www.thieme-connect.de/products die Seite von retten! auf und klicken Sie beim jeweiligen Artikel auf „Zusatzmaterial“.

\section{Kernaussagen}

- Lebensbedrohliche Extremitätenblutungen sind selten, müssen jedoch schnell erkannt und aggressiv therapiert werden.

- In diesem Fall ist die Blutung vor der weiteren Versorgung lebensbedrohlicher Störungen zu stillen ( $<\mathrm{C}>\mathrm{ABCDE})$.

- Neben dem manuellen Abdrücken und dem Druckverband steht mit dem Tourniquet eine effektive Maßnahme zur Blutstillung zur Verfügung.

- Üben Sie Handhabung und Anlage des Tourniquets regelmäßig.

- Das Nutzen-Risiko-Verhältnis eines Tourniquets ist sehr günstig, schwerwiegende Komplikationen sind selten. Die Hauptgefahr besteht in der venösen Stauung und damit der Akzentuierung der Blutung bei ungenügend festem Sitz. 


\section{Schwere Blutungen an Extremitäten So lassen sie sich stoppen}

1

A

Wussage zu kritischen Blutungen stimmt?

Extremitätenverletzungen mit kritischen Blutungen sind im Rettungsdienst häufig anzutreffen.

B $\quad 80 \%$ der katastrophalen Blutungen entfallen auf Extremitätenblutungen.

C Der Volumenersatz mit großen Mengen Infusionsflüssigkeit verursacht keine weiteren Probleme.

D Eine Gerinnungsaktivierung kann durch den Verbrauch an Gerinnungsfaktoren zu einer Gerinnungsstörung führen.

E Ein Mangel an Erythrozyten ist für die Gerinnung unerheblich.

\section{2}

Patienten mit gerinnungshemmender Begleitmedikation sind im Rettungsdienst immer seltener anzutreffen.

B Rivaroxaban ist ein orales Antikoagulans.

C Die Wirkung von Antikoagulanzien und Thrombozytenaggregationshemmern lässt sich präklinisch leicht aufheben.

D Gerinnungshemmende Medikamente haben für die präklinische Versorgung von Blutungen keine Bedeutung.

E Phenprocoumon gehört zu den Thrombozytenaggregationshemmern.

Sie wollen eine starke Blutung am Bein mittels Tourniquet stillen. Es handelt sich um eine offene Fraktur direkt unterhalb des Knies. Wo sollten Sie das Tourniquet anlegen?

A am Oberschenkel möglichst weit proximal

B $\quad 5-10 \mathrm{~cm}$ distal der Verletzung

C auf dem Kniegelenk

D am Oberschenkel möglichst weit distal

E direkt über der Fraktur, da sich eine Anlage auf Höhe des Gelenks verbietet

Was ist keine Indikation zur Anlage eines Tourniquets?

Patient, der sich in suizidaler Absicht die Pulsadern eröffnet hat, starke Blutung, in der Wohnung riecht es nach Gas

B stark blutende Oberarmverletzung, die durch Ersthelfer mittels Druckverband versorgt wurde. Dieser ist leicht blutig durchgeschlagen.

C ersteintreffender RTW bei einem Verkehrsunfall. Insgesamt 3 Patienten, davon einer mit kritischem Blutverlust aus einer Oberschenkelwunde

D traumatische Amputation eines Oberarms, ca. $15 \mathrm{~cm}$ langer Amputationsstumpf

E stark blutende Ablederungsverletzung des Unterschenkels nach Überrolltrauma

Ein Patient erleidet eine tiefe, stark blutende MotorsägenSchnittverletzung am Unterarm. Er hat keine weiteren Verletzungen, zeigt aber Symptome eines Volumenmangelschocks und reagiert nur noch deutlich verzögert und schwach auf Ansprache (GCS 10). Welches Vorgehen wäre adäquat?

A sofort ein Tourniquet anlegen, weiter nach ABCDE-Schema versorgen

B verletzten Arm hochlagern, zuführende Arterie abdrücken, Druckverband, ggf. Tourniquet anlegen, weiter nach ABCDE-Schema versorgen

C Einlage eines Güdeltubus, Sauerstoffgabe, Verband der Wunde, Volumensubstitution

D sofortige Narkoseeinleitung und Intubation bei schlechtem GCS

E sorgfältige Untersuchung nach ABCDE-Schema und Abarbeiten der einzelnen Punkte
6 Was ist keine Komplikation einer (unsachgemäßen)

Tourniquet-Anlage?

Verstärkung der Blutung

Kompartmentsyndrom

Nervenschäden

Hautläsionen

E Kompressionsfraktur des darunterliegenden Knochens

\section{Welche Aussage ist falsch?}

A Gefäßklemmen bieten eine gute Alternative zu anderen blutstillenden Maßnahmen und sollten daher großzügig eingesetzt werden.

B Tourniquets haben ein günstiges Nutzen-Risiko-Profil.

C Die Hauptgefahr bei der Anlage eines Tourniquets besteht in der Blutungsverstärkung bei zu lockerer Anlage.

D Die Wirkung des Tourniquets sollte insb. nach Kreislaufstabilisierung regelmäßig reevaluiert werden.

E Die Anlage eines Tourniquets muss regelmäßig trainiert werden.

\section{Welche Aussage ist richtig?}

A Eine Blutdruckmanschette als Tourniquet-Ersatz sollte am Oberschenkel i. d. R. mit $250 \mathrm{mmHg}$ aufgepumpt werden.

B Eine Blutdruckmanschette als Tourniquet-Ersatz sollte am Oberarm i. d. R. mit ca. $400 \mathrm{mmHg}$ aufgepumpt werden.

C Der Patient sollte nach Möglichkeit auf dem Knebel des Tourniquets liegen, um ein Lösen dessen zu verhindern.

D Der Knebel des Tourniquets sollte gut sichtbar angelegt werden, um ein unbeabsichtigtes Lösen schnell erkennen zu können.

E Eine Blutdruckmanschette ist keine realistische Alternative zum Tourniquet.

9 Welche Reihenfolge bei der Versorgung einer schwer zu stoppenden Blutung ist korrekt?

Tourniquet -2 . Tourniquet - Druckverband - Reposition

B Druckverband - 2. Druckverband - zuführende Arterie abdrücken - verletzte Extremität hochlagern

C Hochlagern - zuführende Arterie abdrücken - Druckverband - evtl. 2. Druckverband - Tourniquet - Tourniquet fester anziehen - 2. Tourniquet sofort 2 Tourniquets anlegen bei erkennbar kritischer Blutung

E Abdrücken der zuführenden Arterie für 30 min, danach Lösen der Kompression und Kontrolle, ob die Blutung sistiert.

Welche Aussage zur Liegedauer des Tourniquets stimmt?

Tourniquets sollten grundsätzlich so lange wie möglich angelegt werden. Die aktuellen Empfehlungen sehen vor, das Tourniquet nach $3 \mathrm{~h}$ zu lösen.

C Wahrscheinlich werden die neuesten Empfehlungen vorsehen, das Tourniquet bereits nach 30 min wieder zu lösen.

D Die Datenlage zur Liegedauer eines Tourniquets ist eindeutig.

E Bei einer Liegedauer $>2 \mathrm{~h}$ treten Komplikationen nicht vermehrt auf.

\section{cee.thieme.de}

- Sammeln Sie CEE-Punkte unter cee.thieme.de für Ihre Rettungsdienstfortbildung. 1 CEE-Punkt entspricht einer Fortbildungsstunde von 60 Minuten. 